\title{
Manifestations of Nearby Moderate Earthquakes in Schumann Resonance Spectra
}

\author{
M. Hayakawa ${ }^{1,2}$, A.P. Nickolaenko ${ }^{3}$, Y.P. Galuk ${ }^{4}$, and I.G. Kudintseva ${ }^{5}$ \\ ${ }^{1}$ Hayakawa Institute of Seismo Electromagnetics Co. Ltd. (Hi-SEM), The University of Electro- \\ Communications (UEC) Alliance Center \#521, 1-1-1 Kojima-cho, Chofu Tokyo, 182-0026, Japan \\ E-mail: hayakawa@hi-seismo-em.jp \\ ${ }^{2}$ Advanced Wireless \& Communications Research Center (AWCC), UEC, 1-5-1 Chofugaoka, Chofu \\ Tokyo, 182-8585, Japan E-mail: hayakawa@hi-seismo-em.jp \\ ${ }^{3}$ A.Ya. Usikov Institute for Radio-Physics and Electronics, National Academy of Sciences of the Ukraine, \\ 12, Acad.Proskura St., Kharkov, 61085,Ukraine E-mail: sasha@ire.kharkov.ua \\ ${ }^{4}$ Department of Mathematics and Computer Sci., Saint-Petersburg State University, 35, University Ave., \\ Peterhof, Saint-Petersburg, 198504, Russia E-mail: j.galuk@spbu.ru \\ ${ }^{5}$ Department of Mathematics and Informatics, V.N. Karazin Kharkov State University, 4, Svobody Square, \\ Kharkov, 61077, Ukraine E-mail: kudintseva@karazin.ua
}

\begin{abstract}
The purpose of this paper is to study theoretically the impact of nearby earthquakes (EQs) on the power spectra of global Schumann resonance (SR) using a numerical model of the ELF (extremely low frequency) radio wave scattering by a localized non-uniformity of the air conductivity profile likely to appear before an EQ. The seismogenic non-uniformity is introduced above the moderate EQ with magnitude $\mathrm{MEQ}=5.5$. The disturbance is characterized by the downward shift of the whole regular conductivity profile, it is axially symmetric, and it varies along the radius from the EQ epicenter based on the Gaussian law. The ELF station is assumed to be located at our Nakatsugawa observatory (Japan). The non-uniformity is assumed to vary its position at distances of $0,100,200,300,400$, and $500 \mathrm{~km}$ from the observing station in the eastern direction. Three positions of SR source (the global thunderstorm centers) are considered: Asia, Africa, and America. The electromagnetic problem is solved with the help of the complex characteristic electric and magnetic heights relevant to the air conductivity profiles. These heights are found by solving the Riccati equation and are substituded into the two-dimensional telegraph equations (2DTE) for computing both the vertical electric and two orthogonal horizontal magnetic fields in the Earth-ionosphere cavity. To single out the spectral modifications at particular frequencies, we compare the power spectra of the regular and the disturbed cavities. The perturbation is found to result in the overall elevation of SR spectra (especially in the electric field). Some dependence is obtained of the field modifications versus distance to the EQ
\end{abstract}


hypocenter. Finally we will try to discuss the relevance of the present numerical results with the corresponding SR observational results during nearby EQs.

Keywords: Seismogenic non-uniformity in the mesosphere, Schumann resonance (SR), Spectral modifications vs distance to $E Q$.

\section{Introduction}

Electromagnetic effects of seismic activity have been extensively investigated during the last three decades (e.g., Pulinets and Boyarchuk, 2004; Molchanov and Hayakawa, 2008; Surkov and Hayakawa, 2014; Hayakawa, 2015; Sorokin et al., 2015; Ouzounov et al. (Eds), 2018; Sorokin et al., 2020; Lizunov et al., 2020), and it is recently recognized that those electromagnetic phenomena do appear prior to an earthquake (EQ). Those seismogenic phenomena can be classified into two categories: one is the direct effect such as electromagnetic radiation from the lithosphere (or atmosphere) in a wide frequency range, and the other is indirect effect such as the seismogenic effects appearing in the atmosphere and the ionosphere (e.g., Uyeda et al., 2008; Hayakawa and Hobara, 2010).

Recently Hayakawa et al. (2020) have published an extensive review only on the first category of those seismogenic effects especially in lower frequency ranges, ULF (ultra low frequency)/ELF(extremely low frequency)/VLF(very low frequency). In the ELF range there is a famous phenomenon of Schumann resonances (SRs) with resonance frequencies around $8 \mathrm{~Hz}$ (fundamental mode, $\mathrm{n}=1), 14 \mathrm{~Hz}(\mathrm{n}=2), 20 \mathrm{~Hz}$ $(n=3)$ etc, which are driven by the global thunderstorm activity (Bliokh et al., 1980; Sentman, 1995; Nickolaenko and Hayakawa, 2002, 2014). Considerable attention has been recently directed to manifestations of seismic activity in the power spectra of SRs (Hayakawa et al., 2005; Nickolaenko et al., 2006; Ohta et al., 2006, 2009; Ouyang et al., 2013; Gazquez et al., 2017; Christofilakis et al., 2019; Florios et al., 2019).

To our knowledge, Maki and Ogawa (1983) were the first that have studied the EQ effect on SRs. However, Hayakawa et al. (2005) found a very convincing seismogenic effect in SRs observed in Japan for a huge EQ in Taiwan (Chi-chi EQ) in such a way that the intensity of the SR in Japan is strongly enhanced at the fourth harmonic and also a significant shift in its peak frequency. This study has suggested a potential tool of SR to monitor the EQ effect. Their case study was furthermore confirmed statistically on the basis of long-term (6 years) observational data (Ohta et al., 2006). As the explanation 
of those anomalies, they have proposed a model of wave interference between the direct wave from a lightning source (American) and the wave scattered by the ionospheric perturbation generated over the EQ epicenter in Taiwan (Hayakawa et al., 2005; Nickolaenko et al., 2006). Similar anomalous SR phenomena associated with different distant EQs have been observed by Hayakawa et al. (2008) as observed in Japan for another Taiwanese EQ, and by Ouyang et al. (2013) as observed in China for the 2011 Tohoku EQ. As a rule, powerful EQs were addressed whose electromagnetic signatures were detected at a distance of a few $\mathrm{Mm}(1 \mathrm{Mm}=1,000 \mathrm{~km})$.

In the present paper, as a further extension of our previous works on distant EQs (e.g., Hayakawa et al., 2005), we pay attention to nearby EQs, i.e. the situation when the EQ epicenter becomes closer to an ELF observing station. We model disturbances in SR spectra relevant to modest EQs with tentative magnitude of 5.5, which occurs at a distance of a few hundred $\mathrm{km}$ from the ELF observing station. To construct the seismogenic non-uniformity, we modify the realistic vertical profile of atmospheric conductivity (Kudintseva et al., 2016; Nickolaenko et al., 2016, 2017, 2018a, b; Galuk et al., 2015, 2019) by shifting this regular profile downward as a whole.

Then, we derive the complex characteristic electric and magnetic heights in the ELF band and the corresponding frequency dependence of the complex propagation constant of ELF radio waves both in the regular and in the non-uniform cavities by using the full wave solution technique in the form of Riccati equation. The seismogenic disturbance modifies the characteristic heights in an isolated area and modifications have the Gaussian radial dependence. Both the vertical electric and two orthogonal horizontal magnetic field components are computed using the two-dimensional telegraph equations (2DTE) in the absence and in the presence of a non-uniformity. These data provide expected modifications in the SR power spectra at varying distances from the EQ center. We will summarize the major theoretical results, and then we will discuss these computational results with reference to previous SR observational facts for nearby EQ events by Maki and Ogawa (1983), Ohta et al. (2009) and Christofilakis et al. (2019). Finally we will make the future suggestion of SR observations and analysis.

\section{Modeling of ionosphere disturbance over the EQ center}

The lower ionosphere is known to shift downward prior to an EQ, which was validated by previous SR works (Hayakawa et al., 2005; Nickolaenko et al., 2006) and also by subionospheric VLF studies 
(Hayakawa et al., 1996; Yoshida et al., 2008). The plots in Fig. 1 illustrate the regular conductivity profile of atmosphere (black smooth curve) (Nickolaenko et al., 2016, 2017, 2018a,b) and the profile at the center of seismogenic non-uniformity (the red line with triangles). The logarithm of conductivity (S/m) is plotted along the abscissa and the ordinate shows the altitude above the ground surface in $\mathrm{km}$. The disturbed conductivity profile we use here is associated with an overall downward uniform displacement of conductivity profile by $16.5 \mathrm{~km}$ with taking into account of our moderate EQ magnitude of 5.5. The disturbance might be a consequence of lithosphere-atmosphere-ionosphere coupling (Molchanov and Hayakawa, 2008). This coupling problem is of the utmost concern to the scientists working on seismoelectromagnetics, even though there have been proposed a few possible agents in the lithosphere (e.g., emanation of radioactive gases (Pulinets and Ouzounov, 2011; Sorokin et al., 2015), impact of atmospheric gravity waves on the mesospheric conductivity (Hayakawa et al., 2011; Yang et al., 2019; Yang and Hayakawa, 2020), positive hole carriers (Freund, 2013) and others (Sorokin et al., 2015)). However, we will not concentrate on these intriguing processes, since our goal of this paper is to answer theoretically the following question: Can a moderate "nearby" EQ cause any noticeable modifications in the power spectra of SR or not? Is there any difference in perturbed characteristics of SR power spectra between the horizontal magnetic field components and vertical electric field component? Because there have been no theoretical expectations on the change in SR spectra when an EQ happens closer to the EQ epicenter.

From the viewpoint of ELF propagation theory in the Earth-ionosphere cavity, the non-uniformity modifies the conductivity profiles simultaneously in the region of the lower (electric) characteristic height of ionosphere $\mathrm{hC} \sim 55 \mathrm{~km}$ and in the vicinity of the upper (magnetic) characteristic height of ionosphere hL 95 km (Greifinger and Greifinger, 1978; Nickolaenko and Hayakawa, 2002; Mushtak and Williams, 2002). Such a disturbance must simultaneously alter the power spectra of the both vertical electric and horizontal magnetic field components.

The horizontal size (characteristic radius) $\rho_{E Q}$ of the area occupied by the seismogenic ionosphere nonuniformity depends on the EQ magnitude (Dobrovolsky et al., 1979; Ruzhin and Depueva, 1996), and we use the following empirical formula by Ruzhin and Depueva (1996):

$$
\rho_{E Q}=\exp \left(M_{E Q}\right)
$$


Here the radius $\rho_{E Q}$ is measured in $\mathrm{km}$, and $M_{E Q}$ is the EQ magnitude. It is clear that $\rho_{E Q}=244.7 \mathrm{~km}$ when $M_{E Q}=5.5$.

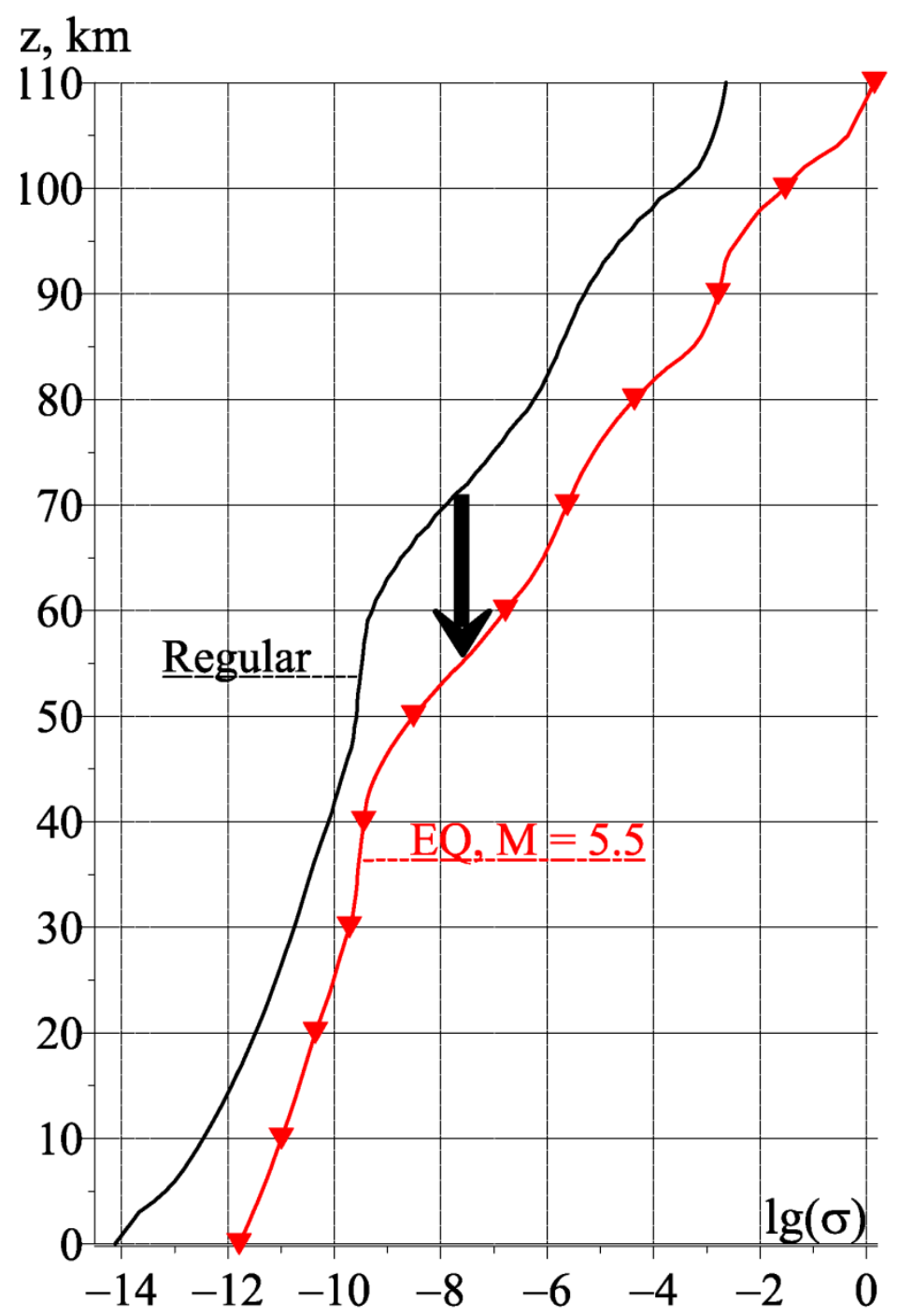

Figure 1: Vertical profiles of atmosphere conductivity above the EQ epicenter

Modifications of atmosphere conductivity reduce the characteristic two heights $h_{C}$ and $h_{L}$. These are the functions of distance from the EQ epicenter. We suggest that the disturbance is axially symmetric, and it changes along the radius in accordance with the Gaussian law:

$$
\delta h=\Delta h \cdot \exp \left(-r_{E Q}^{2} / \rho_{E Q}^{2}\right)
$$


Here $r_{E Q}$ is the distance from the EQ epicenter, and $\Delta h$ is the maximum perturbation of the electric or the magnetic characteristic heights above the EQ center, which is relevant to plots of Fig. 1.

\section{Solution of the problem: ELF scattering problem}

Since the pre-EQ activity generates a localized non-uniformity at the upper boundary of the Earthionosphere cavity, its effect must decrease with the distance between the observer and the EQ epicenter. The anomalies in the power spectra of SR from the wave interference of direct wave coming to the observer from the global thunderstorms and the wave reflected from the non-uniformity above the EQ. This means that the effect will depend on the position of the field source relative to the observer, on the observer-EQ distance, and on the signal frequency.

We present the problem geometry. The ELF observer is assumed to be located at our Nakatsugawa observatory (Japan) (geographic coordinates $35.42^{\circ} \mathrm{N}$ and $137.53^{0} \mathrm{E}$ ) (Ohta et al., 2006, 2009). The perturbation occupies various positions at distances $\mathrm{D}=0,100,200,300,400$, and $500 \mathrm{~km}$ from the observer tentatively to the east (but this direction does not influence later results). Since SR signal is driven by radio emission from the global thunderstorms (Nickolaenko and Hayakawa, 2002), we perform computations for the thunderstorms concentrated in one of three major global thunderstorm centers. The following positions of the field source are considered: Asia $\left(0^{0} \mathrm{~N}\right.$ and $\left.105^{\circ} \mathrm{E}\right)$, Africa $\left(0^{0} \mathrm{~N}\right.$ and $\left.25^{0} \mathrm{E}\right)$, and America $\left(0^{0} \mathrm{~N}\right.$ and $\left.75^{\circ} \mathrm{W}\right)$ (Nickolaenko and Hayakawa, 2014).

The details of the following full-wave solutions have already been described in details in Nickolaenko et al. (2018a,b) and Galuk et al. (2019), and so we will describe only the essential points.

\subsection{Full-wave solution}

The model of Earth-ionosphere cavity incorporates the perfectly conducting spherical Earth surrounded by a poorly conducting air shell bounded by the horizontally stratified ionosphere plasma. The relative dielectric constant of air above the ground is equal to: $\varepsilon(r)=1+i \frac{\sigma(r)}{\omega \varepsilon_{0}}$, where $r$ is the radius vector in the spherical coordinate system $(r, \theta, \varphi)$ with its origin at the Earth's center. The electromagnetic problem for an isotropic vertically inhomogeneous ionosphere is solved in the following steps. After writing Maxwell's equations for the electric and magnetic fields and introducing the appropriate scalar Debye 
potential, one obtains the Helmholtz equation for the TM-wave (E-wave), since only this wave propagates at ELF. This allows us to obtain the standard equation for the radial function:

$$
\left\{\frac{d^{2}}{d r^{2}}+k^{2} \varepsilon(r)-\frac{n(n+1)}{r^{2}}-\sqrt{\varepsilon(r)} \frac{d^{2}}{d r^{2}}\left[\frac{1}{\sqrt{\varepsilon(r)}}\right]\right\} r R(r)=0
$$

The solutions to equation (3) are expressed in terms of spherical Hankel functions $h_{n}^{(1)}(k r \sqrt{\varepsilon})$ and $h_{n}^{(2)}(k r \sqrt{\varepsilon})$.

The electromagnetic problem is formulated and solved using a system of thin horizontal slabs of constant $\varepsilon$. The waves are considered traveling upward and downward in each slab. The continuity boundary conditions hold at each interface for the tangential field components. Thus, a system of linear algebraic equations is obtained for the amplitudes of the waves in the layers. One obtains the system of $2 L$ equations for $L$ layers. Such a treatment is regarded as the full-wave solution (e.g., Wait, 1970).

Application of a linear system of algebraic equations meets a problem when treating SR or ELF radio propagation. The difficulty is associated with too small air conductivity in the troposphere and stratosphere. The linear system becomes degenerate as a result (the equations for different strata become indistinguishable in the lower atmosphere). To avoid this problem, one must either use rather thick lower layers (up to $30 \mathrm{~km}$ ) or reformulate the solution to the Riccati equation. This more convenient variant of solution exploits the surface impedance $\delta(r)$ being the ratio of horizontal electric and magnetic fields at each boundary. The problem for the surface impedance is reduced to the non-linear differential equation of the first order (Riccati equation) (see, e.g., Wait, 1970; Bliokh et al., 1977; Galuk et al., 2015; Kudintseva et al., 2016). Its solution is constructed numerically using an iterative procedure. This equation has the form:

$$
\frac{d}{d r} \delta(r)-i k \varepsilon(r) \delta^{2}(r)+i k+\frac{v(v+1)}{i k r^{2} \varepsilon(r)}=0 .
$$

Here $\delta(r)$ is the varying surface impedance, $v(\omega)$ is the sought complex propagation constant of ELF radio wave, $\omega=2 \pi f$ is the circular wave frequency, $k=\omega / c$ is the wave number in the free space, $c$ is the light velocity, and $r$ is the radius of the spherical coordinate system $(r, \theta, \varphi) ; \varepsilon(r)$ is the complex dielectric constant of the medium varying with height, and $\varepsilon_{0}$ is the dielectric constant of free space. 
The operator of the problem is sought in the height range from zero to infinity, and its eigen-value is $\lambda=v(v+1)$. At the perfectly conducting ground, the surface impedance $\delta(a)=0$, where $a$ denotes the Earth's radius. This is the first boundary condition for Eq. (4).

ELF radio waves of frequency $f$ penetrate into the ionosphere and rapidly attenuates there, so that the plasma properties above some altitude $r_{1}$ have a minor impact on the sub-ionospheric radio propagation, and this altitude depends on the frequency. However, it does not exceed the $110 \mathrm{~km}$ level in the SR band: $r_{1}=a+110 \mathrm{~km}$. Therefore, one may suggest that the ionosphere is vertically uniform above $110 \mathrm{~km}$ altitude, and the surface impedance at $r_{1}$ is equal to $\delta\left(r_{1}\right)=\left[\varepsilon\left(r_{1}\right)\right]^{-1 / 2}$, where $\left|\varepsilon\left(r_{1}\right)\right|=$ const >>1. This is the second boundary condition for Eq. (4).

The problem of finding the complex eigen-value is reduced to solving the equation

$$
\delta(a ; \lambda)=0
$$

with respect to the parameter $\lambda$. The $\delta(a ; \lambda)$ function is found by numerical integration of Eq. (4) from the top $r=r_{1}$ to the bottom $r=a$. The roots of equation are found by iterations from initial value $v=k a$.

Let $\lambda^{m}$ be the $m$-th iteration to the eigen-value $\lambda$. Then, the next, $(m+1)^{\text {th }}$ iteration is obtained by the Newton's procedure:

$$
\lambda^{m+1}=\lambda^{m}-\frac{\delta\left(a ; \lambda^{m}\right)}{\frac{\partial}{\partial \lambda} \delta\left(a ; \lambda^{m}\right)} .
$$

After obtaining the $(m+1)^{\text {th }}$ iteration, the downward integration of Eq. (4) is repeated with the new eigen-value resulting in the new surface impedance at the ground. The iteration procedure stops when the relative difference between the new and the previous eigen-value is below $10^{-7}$. The derivative $\frac{\partial}{\partial \lambda} \delta(a ; \lambda)$ is found simultaneously with the $\delta(r)$ function by integrating the differential equation for the function $\delta_{1}(r)=\frac{\partial}{\partial \lambda} \delta(r, \lambda)$. The relevant equation and the condition at $r=r_{1}$ are obtained by differentiating Eq. (4) with respect to the parameter $\lambda$ :

$$
\frac{d}{d r} \delta_{1}(r)-2 i k \varepsilon(r) \delta(r) \delta_{1}(r)+\frac{1}{i k r^{2} \varepsilon(r)}=0
$$




$$
\delta_{1}\left(r_{1}\right)=0 .
$$

The upper height $r_{1}$ is estimated by using the exhaustive search when solutions are constructed for a series of $r_{1}$ values. Finally, the height $r_{1}=110 \mathrm{~km}$ was chosen.

The anisotropy of ionosphere is unimportant in the SR band since the height interval is characterized by the dominance of collision frequency (Nickolaenko and Hayakawa, 2002). Accounting for the tensor value of air conductivity is necessary either at $\operatorname{VLF}(f \geq 3 \mathrm{kHz})$ or in the band $f<3 \mathrm{~Hz}$ of ionosphere Alfven resonance (IAR) (Surkov and Hayakawa, 2014).

Before computing the field components, we must derive the normalizing integrals. These are obtained using the following relation:

$$
N^{0}=i k a^{2} \frac{\partial}{\partial \lambda} \delta(a ; \lambda)=i k a^{2} \delta_{1}(a)
$$

The normalizing integral $N^{0}$ allows obtaining the lower characteristic height of the Earth-ionosphere cavity:

$$
h_{C}(f) \cong \int_{0}^{\infty} \frac{d h}{1-i \sigma(h) / \omega \varepsilon_{0}}=i k a^{2} \delta_{1}(a)
$$

The lower characteristic height is a complex quantity regarded as the "capacitance", or the "electric", height of the Earth-ionosphere system:

$$
h_{\mathrm{C}}(\theta)=\frac{1}{E_{r}(a, \theta)} \int_{a}^{\infty} E_{r}(r, \theta) d r .
$$

It is linked to the capacitance elements of the two-dimension RLC circuit modeling the Earthionosphere cavity.

The upper, magnetic characteristic height $h_{L}(\theta)=\frac{1}{H_{\varphi}(a, \theta)} \int_{0}^{\infty} \frac{r}{a} H_{\varphi}(r, \theta) d r$ is found by using the relation:

$$
v(v+1)=h_{L} / h_{C}
$$

The heights $h_{C}$ and $h_{L}$ are substituted into the 2D (two dimensional) telegraph equation. 


\subsection{D telegraph equation (2DTE)}

Computations using the $2 D T E$ technique exploit the partial differential equation for the scalar function $u(\theta, \varphi)$ being the voltage between the ground and the lower ionosphere at the point with coordinates $(\theta$, $\varphi)$ :

$$
\frac{h_{L}(\theta, \varphi)}{\sin \theta} \frac{\partial}{\partial \theta}\left(\frac{\sin \theta}{h_{L}(\theta, \varphi)} \frac{\partial u}{\partial \theta}\right)+\frac{h_{L}(\theta, \varphi)}{\sin ^{2} \theta} \frac{\partial}{\partial \varphi}\left(\frac{1}{h_{L}(\theta, \varphi)} \frac{\partial u}{\partial \varphi}\right)+k^{2} a^{2} \frac{h_{L}(\theta, \varphi)}{h_{C}(\theta, \varphi)}\left(u+u_{\text {ст }}\right)=0 \text {, }
$$

The polar axis $\theta=0$ of the spherical coordinate system $(r, \theta, \varphi)$ is directed to the point vertical electric dipole source located at the ground surface. The source function $u_{\mathrm{cT}}$ is defined in the following way:

$$
u_{\mathrm{cr}}=P_{0} \frac{\delta_{D}(\theta)}{2 \pi \varepsilon_{0} a^{2} \sin \theta},
$$

where $P_{0}$ is the dipole moment of the source and $\delta_{D}(\theta)$ denotes the Dirac's delta function.

After obtaining the $2 D T E$ solution $u(\theta, \varphi)$ being the voltage between the ground and the ionosphere, it is possible to turn to physical variables: the vertical electric field $E_{r}$ and the horizontal magnetic field components $H_{S N}$ and $H_{W E}$ :

$$
\begin{aligned}
& E_{r}=\frac{u(\theta, \varphi)}{H_{C}}, \\
& H_{\varphi}=\frac{i}{k a Z_{0} H_{L}} \frac{\partial u}{\partial \theta}, \\
& H_{\theta}=\frac{i}{k a Z_{0} H_{L} \sin \theta} \frac{\partial u}{\partial \varphi}
\end{aligned}
$$

All these fields are the complex functions of frequency since they are the Fourier transforms of the real functions of time. $Z_{0}$ is the free space wave impedance. Usually only the $E_{r}$ component was addressed in the literature, but here we additionally compute the orthogonal magnetic field components $H_{S N}$ and $H_{W E}$. We exploit the universal grid technique (or the method of finite differences) for solving Eq. (13).

The major difficulty associated with the spherical coordinates $(\theta, \varphi)$ consists in the singularities at the poles $(\theta=0$ and $\theta=\pi)$. These might be avoided by an appropriate selection of grid nodes. A grid with the number of nodes $N$ and $M$ correspondingly is superimposed on the 'rectangular' domain of variables $(\theta$, $\varphi$ ). The grid spacing along the variable $\theta$ (co-latitude) is equal to $h_{\theta}=\pi / N$. The step along the $\varphi$ variable 
(the longitude) is correspondingly equal to $h_{\varphi}=\pi / M$. The nodal coordinates of the grid are related to their numbers $\{i, j\}$ by following relations: $\theta_{i}=(i+1 / 2) h_{\theta}, i=\in[0, N-1], \varphi_{j}=j h_{\varphi}, j=\in[0, M-1]$.

The boundary points $i=0$ and $i=N-1$ are separated from the poles exactly by one-half of the grid step $h_{\theta}$ with such nodes along the $\theta$ coordinate. Therefore, it is unnecessary to calculate the coefficients at the singular points.

The first derivatives of the sought function at the nodes are replaced by the finite differences of the first order. The discrete sampling of Eq. (13) provides a closed system of equations for the grid functions $u_{i, j}$ :

$$
\begin{aligned}
& h_{\varphi}^{2} \frac{h_{L}\left(\theta_{i}, \varphi_{j}\right)}{\sin \theta_{i}}\left\{\frac{\sin \left(\theta_{i}+h_{\theta} / 2\right)}{h_{L}\left(\theta_{i}+h_{\theta} / 2, \varphi_{j}\right)}\left(u_{i+1, j}-u_{i, j}\right)-\frac{\sin \left(\theta_{i}-h_{\theta} / 2\right)}{h_{L}\left(\theta_{i}-h_{\theta} / 2, \varphi_{j}\right)}\left(u_{i, j}-u_{i-1 j}\right)\right\}+ \\
& +h_{\theta}^{2} \frac{h_{L}\left(\theta_{i}, \varphi_{j}\right)}{\sin ^{2} \theta_{i}}\left\{\frac{u_{i, j+1}-u_{i, j}}{h_{L}\left(\theta_{i}, \varphi_{j}+h_{\varphi} / 2\right)}-\frac{u_{i, j}-u_{i, j-1}}{h_{L}\left(\theta_{i}, \varphi_{j}-h_{\varphi} / 2\right)}\right\}+h_{\theta}^{2} h_{\varphi}^{2} k^{2} a^{2} \frac{h_{L}\left(\theta_{i}, \varphi_{j}\right)}{h_{C}\left(\theta_{i}, \varphi_{j}\right)} u_{i, j}=0,
\end{aligned}
$$

where $\theta_{i}=(i+1 / 2) h_{\theta}, i=\in[0, N-1]$, and $\varphi_{j}=j h_{\varphi}, j=\in[0, M-1]$

The grid equation is constructed separately in the pole vicinity $\theta=0\left(i=0, \theta_{0}=h_{\theta} / 2\right), j=\in[0, M-1]$ with an account for the expression $\sin \theta \frac{\partial u}{\partial \theta}=0$ at the poles $(\theta=0$ and $\theta=\pi)$. These points are separated from the poles by the $h_{\theta} / 2$ distance:

$$
\begin{aligned}
& 2 h_{\varphi}^{2} \frac{h_{L}\left(h_{\theta} / 2, \varphi_{j}\right)}{h_{L}\left(h_{\theta}, \varphi_{j}\right)}\left(u_{1, j}-u_{0, j}\right)+4 h_{L}\left(\theta_{0}, \varphi_{j}\right)\left\{\frac{u_{0, j+1}-u_{0, j}}{h_{L}\left(\theta_{0}, \varphi_{j}+h_{\varphi} / 2\right)}-\frac{u_{j}-u_{0, j-1}}{h_{L}\left(\theta_{0}, \varphi_{j}-h_{\varphi} / 2\right)}\right\}+ \\
& +h_{\theta}^{2} h_{\varphi}^{2} k^{2} a^{2} \frac{h_{L}\left(\theta_{0}, \varphi_{j}\right)}{h_{C}\left(\theta_{0}, \varphi_{j}\right)} u_{0, j}=0, j=0, \ldots, M-1 .
\end{aligned}
$$

The grid equations acquire the following form in the vicinity of the other pole $(\theta=\pi)\left(i=N-1, \theta_{N-}\right.$ $\left.{ }_{1}=\pi-h_{\theta} / 2, j=\in[0, M-1]\right)$ :

$$
\begin{aligned}
& 2 h_{\varphi}^{2} \frac{h_{L}\left(\theta_{N-1}, \varphi_{j}\right)}{h_{L}\left(\pi-h_{\theta}, \varphi_{j}\right)}\left(u_{N-2, j}-u_{N-1, j}\right)+4 h_{L}\left(\theta_{N-1}, \varphi_{j}\right)\left\{\frac{u_{N-1, j+1}-u_{N-1, j}}{h_{L}\left(\theta_{N-1}, \varphi_{j}+h_{\varphi} / 2\right)}-\frac{u_{N-1, j}-u_{N-1, j-1}}{h_{L}\left(\theta_{N-1}, \varphi_{j}-h_{\varphi} / 2\right.}\right\}+ \\
& +h_{\theta}^{2} h_{\varphi}^{2} k^{2} a^{2} \frac{h_{L}\left(\theta_{N-1}, \varphi_{j}\right)}{h_{C}\left(\theta_{N-1}, \varphi_{j}\right)} u_{N-1, j}=P_{0} \frac{k^{2}}{\pi \varepsilon_{0} h_{\theta}^{2}} \frac{h_{L}(\pi, 0)}{h_{C}(\pi, 0)} .
\end{aligned}
$$

We exploit the cyclic properties of variable $\varphi$ in the above equations: the index should be replaced by $M-1$ when it reaches the value of -1 , and it must be replaced by 0 when arriving at $M$. 
Thus, we obtain the closed system of $N \times M$ linear algebraic equations (18) - (20) for the $N \times M$ unknowns $u_{i, j}$. We use $N=199$ and $M=150$ in particular computations.

Equations of the above type are often met in applications, and there are many methods for their solution. We have chosen the direct method of solution called block tri-diagonal matrix algorithm (Samarskii, 2001). This method solves the problem in the following way: the vectors $\vec{Y}_{i}, i=0, \ldots, N$ are sought that satisfy the equation:

$$
A_{i} \vec{Y}_{i+1}-B_{i} \vec{Y}_{i}+C_{i} \vec{Y}_{i-1}=-\vec{F}_{i}, 1 \leq i \leq N-1
$$

and the boundary conditions:

$$
\begin{aligned}
& A_{0} \vec{Y}_{1}-B_{0} \vec{Y}_{0}=-\vec{F}_{0}, \\
& B_{N} \vec{Y}_{N}+C_{N} \vec{Y}_{N-1}=-\vec{F}_{N},
\end{aligned}
$$

where $A_{i}, B_{i}$, and $C_{i}, i=0, \ldots, N-1$ are the square matrices. The solution has the following form:

$$
\vec{Y}_{i-1}=X_{i} \vec{Y}_{i}+\vec{Z}_{i}, \quad i=N, N-1, \ldots, 1,
$$

where $X_{i}$ and $\vec{Z}_{i}$ are the matrices and vectors sought. Initially, the direct sweep of matrix is performed:

$$
\begin{aligned}
& X_{i+1}=\left(B_{i}-C_{i} X_{i}\right)^{-1} A_{i}, \quad X_{1}=B_{0}^{-1} A_{0}, \\
& \vec{Z}_{i+1}=\left(B_{i}-C_{i} X_{i}\right)^{-1}\left(C_{i} \vec{Z}_{i}+\vec{F}_{i}\right), \\
& \vec{Z}_{1}=B_{0}^{-1} \vec{F}_{0}, \quad i=\{1,2, \ldots, N-1\}
\end{aligned}
$$

Afterwards, the inverse sweep is made that provides the solution:

$$
\begin{aligned}
& \vec{Y}_{N}=\left(B_{N}-C_{N} X_{N}\right)^{-1}\left(C_{N} \vec{Z}_{N}+\vec{F}_{N}\right), \\
& \vec{Y}_{i-1}=X_{i} \vec{Y}_{i}+\vec{Z}_{i}, \quad i=N, N-1, \ldots, 1 .
\end{aligned}
$$

A more detailed description of this algorithm might be found in the book by Samarskii (2001). The major advantage of this method is a substantial gain in the computational resources. The number of calculations increases as $M^{3}$ being the number of partitions along the longitude (the amount of memory allocated to arrays is proportional to $M^{2}$ ). The fast increase originates from conversion of the complex matrix of dimension $M$. The number of arithmetic operations and the necessary memory increase as $N$ 
being the partition number along the latitude. In real computations, one can choose the $M$ parameter several times smaller than the number $N$.

It should also be noted that, owing to the numbering of grid nodes, all the arrays of the right-hand parts are equal to zero $\vec{F}_{i}=0$ except the last one $\vec{F}_{N}$. This allows ignoring the intermediate arrays $\vec{Z}_{i}$. One may observe from Eq. (18) that the arrays of coefficients $A_{i}$ and $B_{i}$ have the simple diagonal form, and the $C_{i}$ array is of a tri-diagonal form. This simplifies the algebraic operations in formula (24).

\section{Computational results}

Figure 2 illustrates the power spectra of SR for the lightning sources positioned in Asia, Africa, or in America arranged in three lines. It contains nine panels. The left column of panels corresponds to the observer - EQ distance $D=0 \mathrm{~km}$; the middle column shows the data for the observer $-\mathrm{EQ}$ distance $D=$ $300 \mathrm{~km}$; and the right column corresponds to $D=500 \mathrm{~km}$. The abscissa at each panel depicts the signal frequency in the SR band $5-35 \mathrm{~Hz}$. The ordinates show the spectral density in dB. Upper graphs in each panel depict the power spectra of vertical electric field component. The red lines here show spectra of the cavity with seismogenic non-uniformity, and the black lines are the spectra in the regular cavity. As one may observe from the figure, the localized non-uniformity "elevates" the SR spectrum as a whole, and this modification decreases with distance $D$. The lower lines in Fig. 2 frames present the power spectra of horizontal magnetic fields. The violet and blue lines show correspondingly the power spectra of $H_{W E}$ and $H_{S N}$ fields in the non-uniform cavity. The magenta and the brown lines depict relevant power spectra in the uniform cavity. Again, the disturbance "elevates" the SR spectra and more distant EQs provide smaller electromagnetic effects.

Three middle plots in the panels of Fig. 2 illustrate the frequency dependence of deviations in the power spectra of three field components (in $\mathrm{dB}$ ). The orange lines show relative modifications of vertical electric field $E_{r}$, while the green and the black lines correspond to deviations in the power spectra of $H_{W E}$ and $H_{S N}$ field components. These curves clearly show that seismogenic modifications in the orthogonal magnetic fields are coincident and these are found to be smaller than the disturbances in the vertical electric field.

We show the model spectra computed for the field sources concentrated at one of the global thunderstorm centers (point sources) in Fig. 2. Obviously the data for different positions of thunderstorms 
have a coincident feature: the plots relevant to the disturbed cavity are elevated above the spectra pertinent to the uniform Earth - ionosphere cavity. The distinctions in the general outline of SR patterns are caused by different source-observer distances (Nickolaenk and Hayakawa, 2002, 2014). Still the relative modifications of the power spectra remain similar within the vertical columns of frames in Fig. 2.
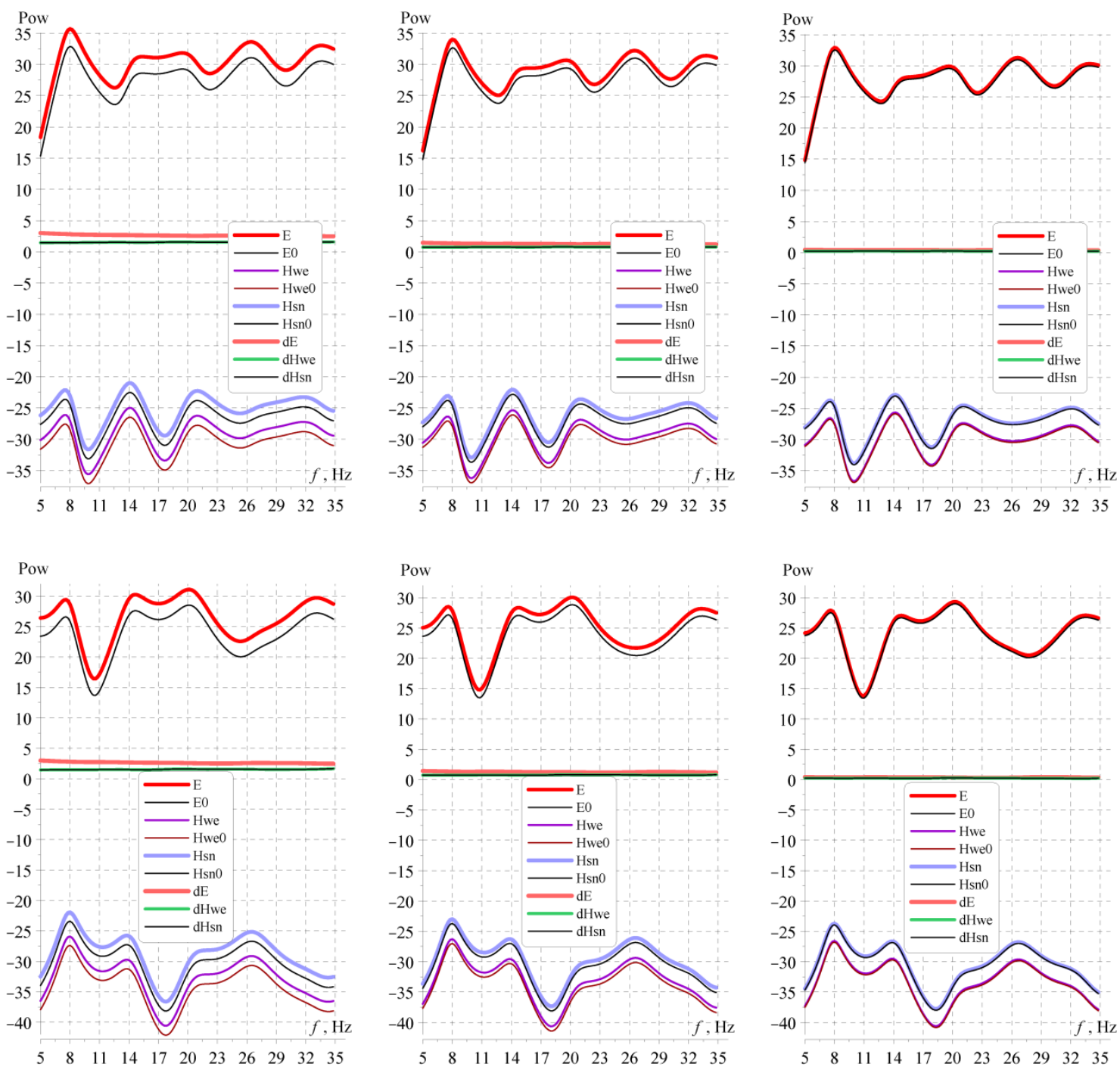

Figure 2: R spectra for Asian, African and American sources and three distances from the EQ epicenter.

Figure 3 contains the maps demonstrating frequency - distance variations of spectral modifications caused by a seismogenic non-uniformity. The abscissa of each panel indicates the signal frequency in the 
$5-35 \mathrm{~Hz}$ band, and the ordinate depicts the observer - EQ distance (D) from 0 to $500 \mathrm{~km}$. The colors (the color scale is shown at the right of plots) indicate the relative field disturbance as defined below:

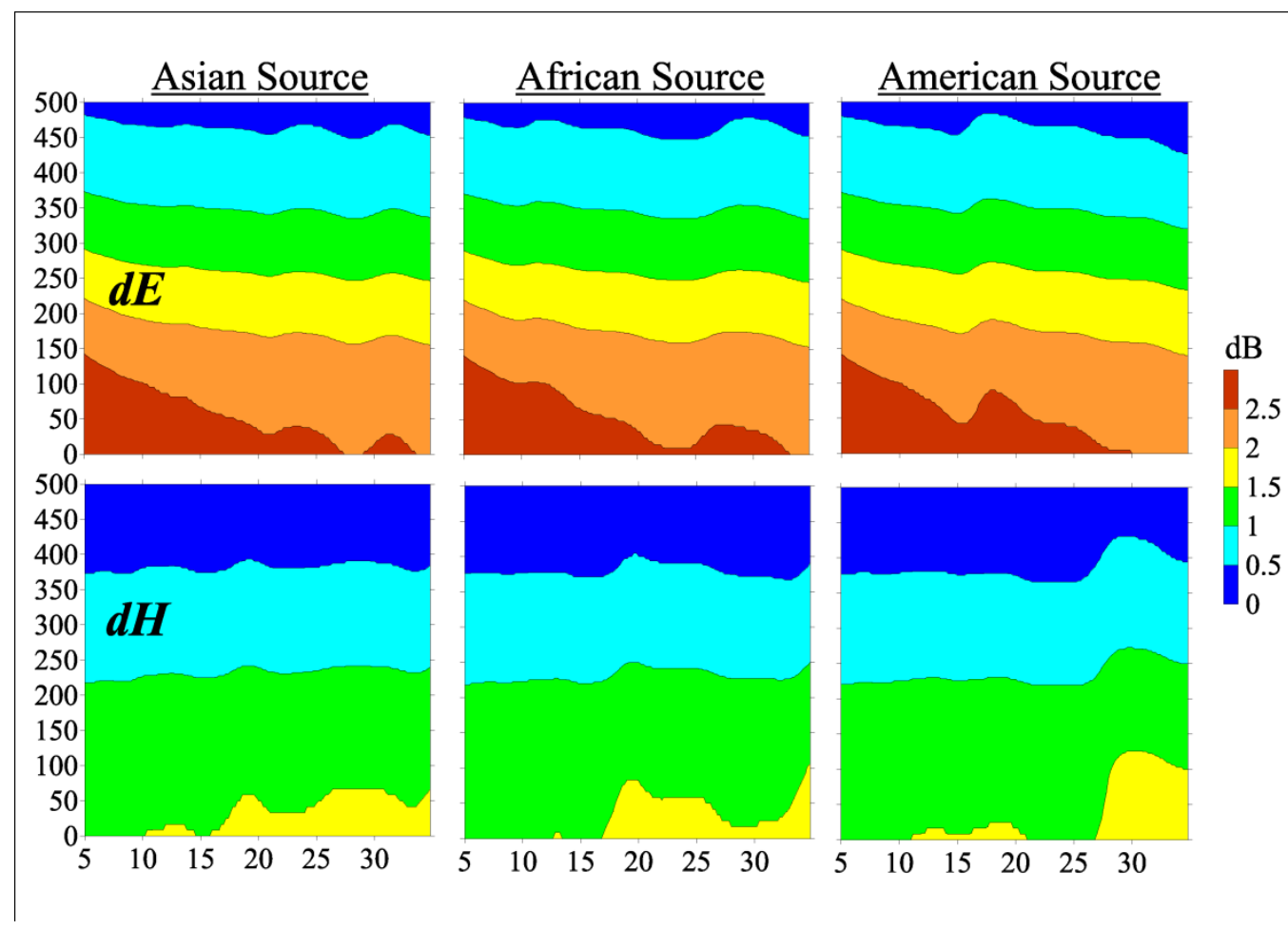

Figure 3: 2D profiles of disturbance (dB) in the power spectra of $E_{r}$ and $H_{W E}$ field components over the frequency distance plane.

$$
\begin{aligned}
& d E=10 \cdot \log \left(\frac{\left\langle\left|E_{r}\right|^{2}\right\rangle}{\left\langle\left|E_{0}\right|^{2}\right\rangle}\right), \\
& d H_{W E}=10 \cdot \log \left(\frac{\left\langle\left. H_{W E}\right|^{2}\right\rangle}{\left\langle\left|H_{W E 0}\right|^{2}\right\rangle}\right), \\
& d H_{S N}=10 \cdot \log \left(\frac{\left\langle\left. H_{S N}\right|^{2}\right\rangle}{\left\langle\left|H_{S N 0}\right|^{2}\right\rangle}\right) .
\end{aligned}
$$

Here $\left\langle\left|E_{r}^{2}\right|\right\rangle,\left\langle\left|H_{W E}\right|^{2}\right\rangle$, and $\left\langle\left|H_{S N}\right|^{2}\right\rangle$ denote the disturbances (dB) in the power spectra of vertical electric and horizontal magnetic field components; $\left\langle\left|E_{0}{ }^{2}\right|\right\rangle,\left\langle\left|H_{W E 0}\right|^{2}\right\rangle$, and $\left\langle\left|H_{S N O}\right|^{2}\right\rangle$ are the power spectra $(\mathrm{dB})$ in the regular Earth - ionosphere cavity. 
The left column of plots in Fig. 3 corresponds to the Asian source; the middle and the right columns show relative disturbances for African and American sources. Figure 3 clearly suggests that impact of the moderate EQ tends to uniformly decrease with the distance between the observer and EQ regardless of the distance to the lightning source.

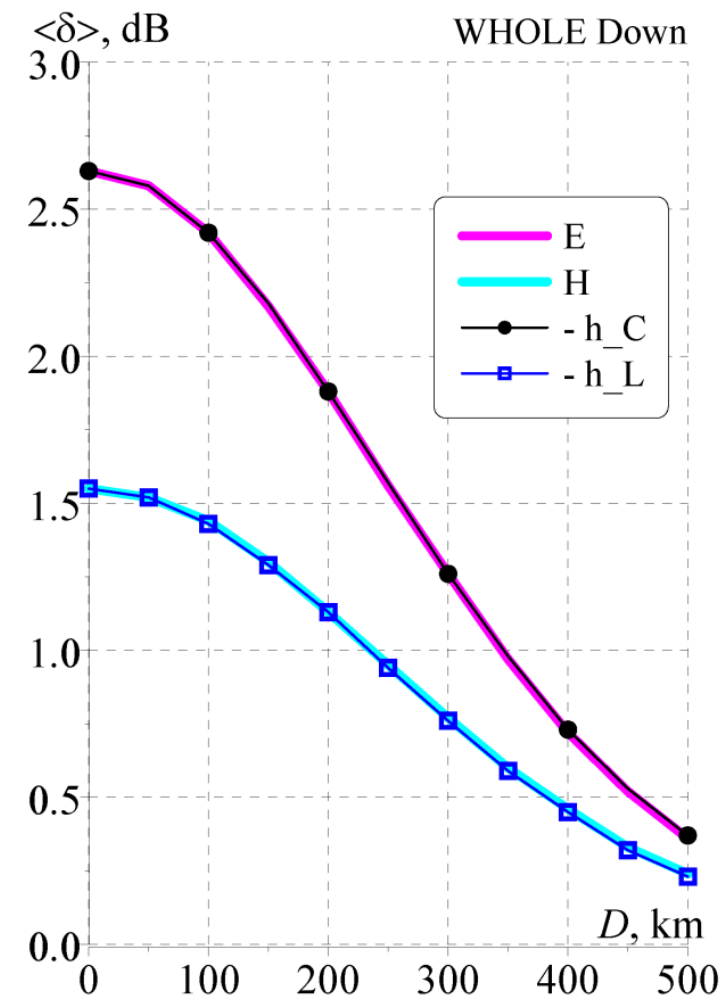

Figure 4: Relative field disturbances and ionosphere characteristic heights above the ELF observer as a function of observer - EQ distance.

Distance variations of the field disturbances averaged over the frequency in the SR band are summarized in Fig. 4. The abscissa here shows the distance from observer to the EQ epicenter. The ordinate refers to the relative modifications of the fields in $\mathrm{dB}$. The red line shows data corresponding to the vertical electric field; the blue and the black line with dots indicate distance variations of the $H_{W E}$ and $H_{S N}$ field components. One may observe a gradual decrease of spectral modifications with the distance from the EQ epicenter, while the seismogenic effect is found to be more pronounced in the vertical electric field component. 
The pattern of distance variations shown in Fig. 4 reminds us of a Gaussian curve (2). To check the validity of this idea, we compare in Fig. 5 variations of SR field power and the relative disturbances (in $\mathrm{dB})$ of characteristic electric $h_{C}$ and magnetic $h_{L}$ heights above the observatory.

The abscissa in Fig. 5 shows the distance $D$ between the observer and EQ epicenter, and the ordinate depicts the relative disturbances in $\mathrm{dB}$. The magenta curve shows the disturbance in the vertical electric field power and the blue line depicts distance variations of disturbance in horizontal magnetic fields. Data were averaged over the frequency band $5-35 \mathrm{~Hz}$. Two additional graphs in Fig. 5 illustrate relative distance variations of disturbance in characteristic heights averaged over the same frequency band:

$$
-\delta h_{C}[d B]=20 \cdot \log \left(\left|\frac{h_{C}}{h_{C 0}}\right|\right)
$$

and

$$
-\delta h_{L}[d B]=20 \cdot \log \left(\left|\frac{h_{L}}{h_{L 0}}\right|\right) .
$$

Here, $h_{C 0}$ and $h_{L 0}$ denote the regular electric and magnetic characteristic heights; $h_{C}$ and $h_{L}$ are the characteristic heights in the non-uniform cavity at the given distance from the EQ epicenter, and the radial dependence of the latter was introduced by Eq. (2).

Figure 5 indicates that the relative disturbance of the spectral density of SR in a close vicinity of the EQ epicenter is driven by the absolute changes of characteristic heights of the air conductivity profiles right above the observatory. Modifications of the electric height cause alterations of the vertical electric field, and disturbance in magnetic characteristic height influences the horizontal magnetic field. From the formal point of view, a decrease in characteristic heights above the ELF observer causes an equivalent increase in the field spectra since the characteristic heights are met in the denominators of formulae describing ELF electromagnetic fields.

\section{Computational results for other perturbed profiles}

The model of seismogenic ionosphere disturbance used in the previous section is based on the downward vertical shift of the whole conductivity profile, which seems to be validated by earlier SR works (Hayakawa et al., 2005; Nickolaenko et al., 2006) and also by VLF studies (Hayakawa et al., 1996; Yoshida et al., 2008). It is found in the previous section that such a modification leads to some noticeable 
increase in the power spectra of both vertical electric and horizontal magnetic field components. But it is clear that other models might be used that were widely discussed in the literature (e.g., Sorokin et al., 2015; Ouzounov et al. (Eds), 2018).

For example, the conductivity profile might be shifted upward. Computations show that the sign of spectral modification will change in this case and the spectral density of field power will decrease both in the vertical electric and the horizontal magnetic fields.

One also may use the modification of profile when the air conductivity remains unchanged in the upper part of mesosphere, while it grows at the heights below $75-80 \mathrm{~km}$ as if this part of profile is shifted downward. This kind of disturbance will be regarded as Bottom Down (BD) model, and the relevant profile is shown in Fig. 5 by blue line. This figure is similar to Fig. 1. The abscissa here depicts the logarithm of air conductivity in $\mathrm{S} / \mathrm{m}$ and the ordinate shows the height above the ground surface. The thick green line shows the regular profile in atmosphere conductivity. The BD model profile is coincident with the regular one above $80 \mathrm{~km}$ altitude. It is coincident with the disturbed profile shown in Fig. 1 below $63 \mathrm{~km}$ altitude. The conductivity abruptly changes between the 63 and $80 \mathrm{~km}$ heights. Such a profile might be suggested when one treats the atmosphere modifications in its lower strata, e.g., some agent emanated from the ground in the seismic area drifts upward in the air increasing its conductivity in the interval up to $60-65 \mathrm{~km}$. Modifications are absent starting from $80 \mathrm{~km}$ altitude.

The alternative modifications regarded as Top Down (TD) model are shown by red line with dots in Fig. 5. The TD model profile is coincident with the regular green curve in the height interval from 0 to 80 $\mathrm{km}$. At $80 \mathrm{~km}$, the air conductivity abruptly increases by almost two orders of magnitude. The TD profiled is coincident with the disturbed profile from Fig. 1 above the $81 \mathrm{~km}$ altitude, and modifications are absent below the $80 \mathrm{~km}$ altitude. This model might correspond to the other disturbances in the atmosphere associated with, say, the ultra-low frequency atmospheric gravitational waves traveling from the ground toward the lower ionosphere. These waves pass though the troposphere and stratosphere strata gaining simultaneously the amplitude due to an exponential decrease in air density with altitude. Starting from $80 \mathrm{~km}$, the atmospheric gravity waves reach the amplitude capable to launch the non-linear effects, which increase the air conductivity. 


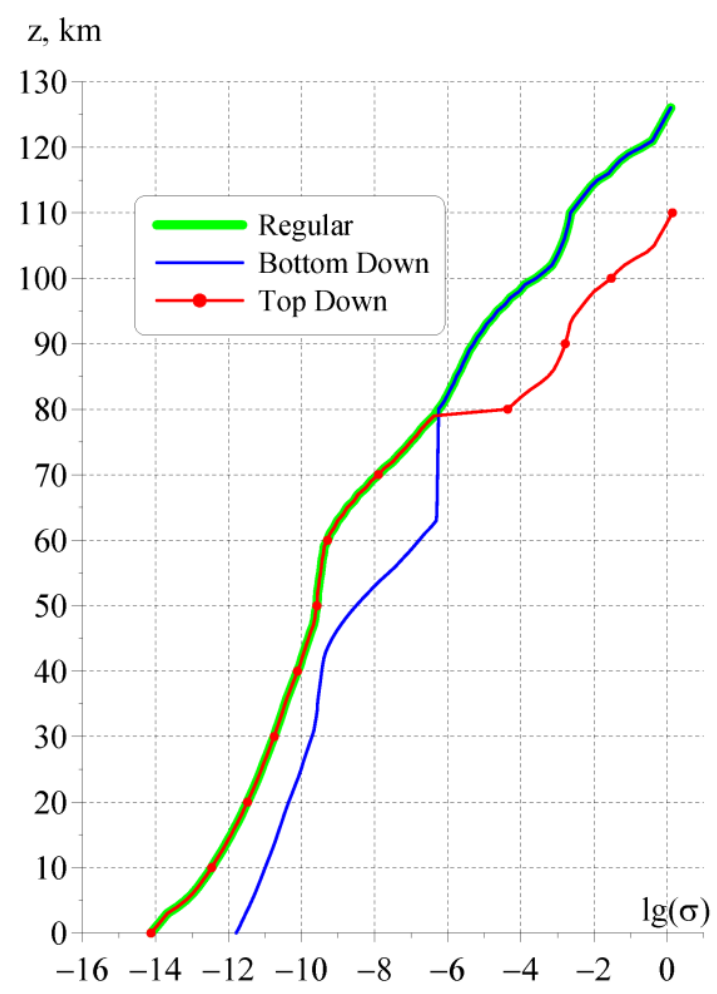

Figure 5: Disturbed profiles of TD and BD types for EQs with magnitude $M_{E Q}=5.5$.

From the viewpoint of radio propagation, the distinction of these BD and TD models from the initial WHOLE model in Sections 3 and 4 is in separate modifications of either electric (lower) or the magnetic (upper) characteristic height of ionosphere. As a result, the electromagnetic properties of the Earth ionosphere cavity are modified in different ways, so that SR spectra might demonstrate the distinctive reactions to seismogenic disturbances.

To save the place, we do not show particular SR spectra relevant to TD and BD profiles. Instead, we demonstrate in Fig. 6 the disturbances in the power spectra averaged over the frequency in SR band. Figure 6 contains two panels. The left one (Fig. 6a) shows the data relevant to the BD model, and the right one (Fig. 6b) corresponds to the TD profile shown in Fig. 5. The abscissas in Fig. 6 depict the distance $D$ between the observer and the EQ epicenter in $\mathrm{km}$, and the ordinates show relative disturbances in the power spectra in $\mathrm{dB}$ defined by Eqs. (26) - (28). 


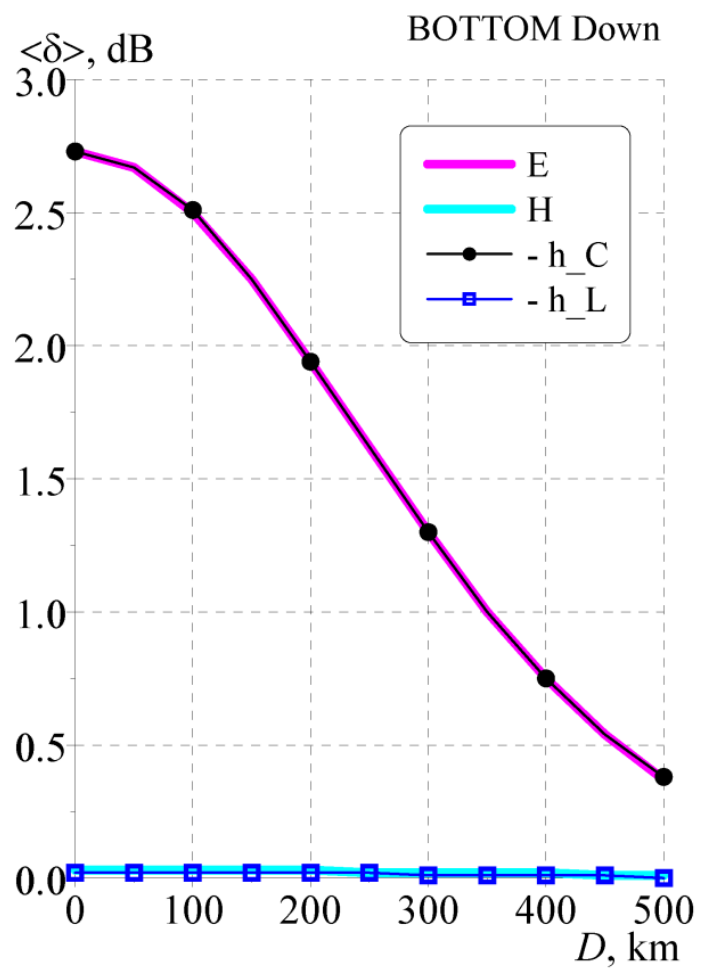

a-BOTTOM Down profile

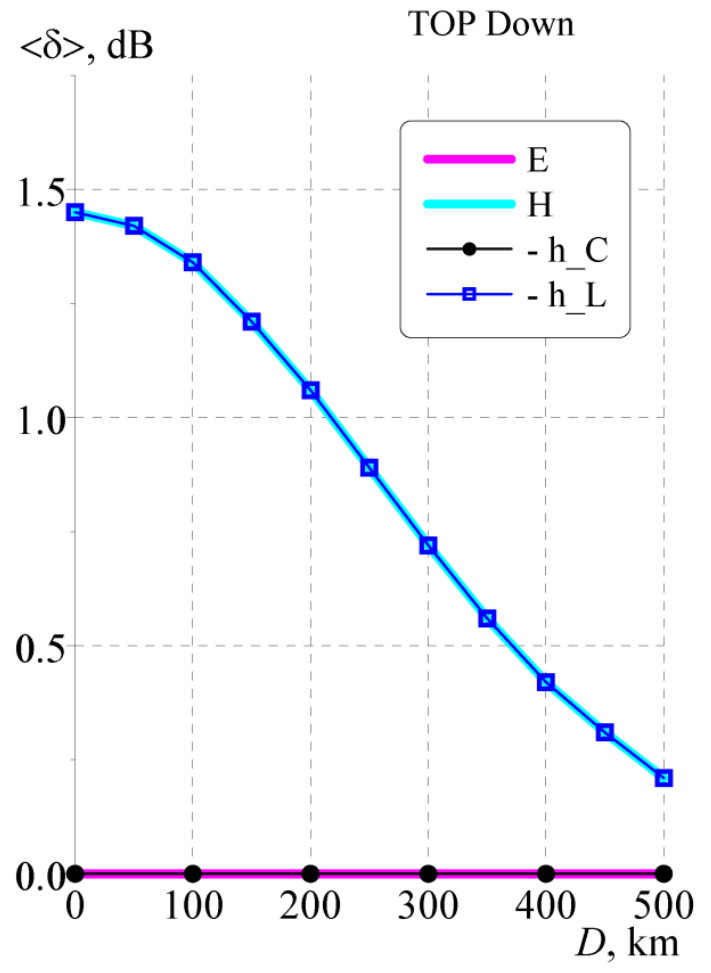

b - TOP Down profile

Figure 6 : Spectral modifications in vertical electric and horizontal magnetic fields caused by the non-uniformities of the TD and BD types for American lightning source.

By comparing Fig. 6a and 6b, one may conclude that different kinds of modifications in conductivity profiles cause distinct spectral disturbances. The BD model is connected with the noticeable changes in the power spectra of vertical electric field and negligible modifications of horizontal magnetic field. The TD model provides the 'opposite' alterations: it modifies the horizontal magnetic field and provides no impact on the electric field. Obviously, the disturbances in the SR power spectra remain remarkably similar to the modifications of characteristic heights above the ELF observatory. We may infer the particular localization of the possible ionosphere modification by analyzing disturbances observed in the SR spectra of vertical electric and horizontal magnetic fields. The presence of modifications exclusively in the electric or in the magnetic fields indicates the predominant alterations of air conductivity in the vicinity of either $h_{C} \sim 55 \mathrm{~km}$ or $h_{L} \sim 95 \mathrm{~km}$ characteristic heights. One may state that the whole 
conductivity profile moves vertically when the disturbances observed in the vertical electric field are twice as big as those in the horizontal.

\section{Summary of computations}

First of all, we can summarize the computational results of the changes in SR power spectra for seismogenic ionospheric perturbations.

(1) Model computations for a perturbation with uniform downward shift in atmospheric conductivity profile in Section 4 indicate that moderate nearby EQs are able to provide noticeable modifications of SR signals. "Elevation" of SR power spectra reaching $1 \mathrm{~dB}$ might be expected in the horizontal magnetic field when the ELF observatory is positioned within the distance $\rho_{E Q}=\exp \left(M_{E Q}\right) \mathrm{km}$ from the epicenter of an EQ with moderate magnitude $M_{E Q}$. Similar modifications in the vertical electric field component might be observed at a distance of about $1.5 \exp \left(M_{E Q}\right)$ and also maximum elevation in the electric field amounts to $3 \mathrm{~dB}$. These perturbations are expected to be more enhanced for EQs with larger magnitude and closer to the ELF observing station.

(2) On the contrary if the perturbation moves upwards, we will have completely the opposite tendency such as the overall decrease in SR power spectra for both the electric and magnetic field components.

(3) The computational results for other perturbed profiles (BD or TD model) in Section 5 suggests that the properties and their comparison of the changes in SR power spectra for the horizontal magnetic fields and vertical electric field might indicate at which altitude the perturbation is generated with special reference to the characteristics heights, electric $\left(\mathrm{h}_{\mathrm{C}}\right)$ and magnetic $\left(\mathrm{h}_{\mathrm{L}}\right)$.

\section{Comparison with observational results and conclusion}

Here we try to compare our computational results for modeled ionospheric perturbations with earlier observations of SR spectra before and after an EQ. As is well known, SR signals propagate in the Earthionosphere cavity in the TEM mode, so that normally we observe simultaneously two horizontal magnetic field components (Bx, By) and one vertical electric field (Ez(or Er in this paper)) (Nickolaenko and Hayakawa, 2002, 2014). However, there are few groups measuring three orthogonal magnetic field components as a higher frequency extension of ULF magnetic field observation (Ohta et al., 2006; Schekotov and Hayakawa, 2017). 
As long as we know, Maki and Ogawa (1973) were the first to have investigated the EQ effect on SRs, in which they dealt with EQs within $200 \mathrm{~km}$ from their ELF station. They used only a ball antenna to measure the Ez component, and they found that the resonance amplitudes increase before several (only inland) EQs with magnitude larger than 3.0. Their analysis was focused on imminent precursors (just a few hours before an EQ), and no information was available for short-term (lead time of a few days to a week) precursors of our main interest. Initially they intended to interpret this phenomenon as the emission of wide-band noise from the lithosphere as the first category of seismo-electromagnetic phenomena as presented in Introduction. Recently it is becoming very evident that there take place clear impulsive ULF/ELF ( $\mathrm{f}=1-10 \mathrm{~Hz}$ ) electromagnetic atmospheric emissions before an EQ (Schekotov et al., 2007, 2013; Ohta et al., 2013; Schekotov and Hayakawa, 2017), so that the first result by Maki and Ogawa (1973) can be explained in terms of this recent emission result. However, based on our computational results in this paper, Maki and Ogawa' result can also be reasonably accounted for in terms of the presence of seismogenic ionospheric perturbation in association with EQs close to their ELF station.

Next we pay attention to a recent paper by Christofilakis et al. (2018), who have tried to find any significant disturbances in SR for the nearby (distance of 200-250 km) EQs in Greece with the measurement of two horizontal magnetic field components (no electric field measurement). They have found that the $3^{\text {rd }}(\mathrm{n}=3)$ mode $(\mathrm{f}=18-25 \mathrm{~Hz})$ is enhanced and at the same time the fundamental mode is suppressed. But this observational behavior seems to be inconsistent with our computational, because our computational result suggests which has yielded the overall enhancement at all modes as long as any change appears. So, unfortunately we cannot give any definite answer to their observational result.

Additionally, in Christofilakis et al. (2018) electromagnetic events with narrow band structure around $21 \mathrm{~Hz}$ overlapped on the conventional SR spectra have been detected before EQs with moderate magnitude around 5. Similar types of line emissions have already been observed before by Ohta et al. (2009) for the nearby (distance of a few hundred $\mathrm{km}$ ) Japanese EQs based on the magnetic field measurement. In the first EQ (Niigata-Chuetsu) the enhancement of the SR $n=3$ mode was detected together with the generation of a signal around $16 \mathrm{~Hz}$ in the By component, and another around $18 \mathrm{~Hz}$ in the Bx component. In the second event (Noto-Hantou EQ) they observed the enhancement of the SR $n=3$ mode was also detected together with a $18 \mathrm{~Hz}$ signal in the $\mathrm{Bx}$ component. It seems obvious that those line emissions are not interpreted in terms of the ionospheric perturbation as presented in this paper. So we have to explore any other mechanism, and one possibility is likely to be the generation of gyrotropic 
waves excited by wide-band waves from below in the ELF band (Sorokin and Hayakawa, 2008; Hayakawa et al., 2010; Sorokin et al., 2015). The frequency of those line emissions is determined as eigen-frequency of gyrotropic waves in a thin layer in the E region, but we need to have wide-band noise from below such as impulsive ULF/ELF noise studied by Schekotov et al. (2007, 2013) and Schekotov and Hayakawa (2017).

As the conclusion, we were not so successful in explaining some anomalous SR observational results by using the computational results in this paper. However, we have to emphasize the potential use of SR observation, not only in the conventional field of study of global lightning activity and global warming (e.g. Williams, 1992; Heckman et al., 1998; Sekiguchi et al., 2006; Price et al., 2007; Satori et al., 2009; Shvets and Hayakawa, 2011; Nickolaenko et al., 2011; Pracser et al., 2019), but also in a new direction to the study of seismo-electromagnetics as suggested in this paper. Already there have been established and in operation so many ELF observatories in the world (e.g. see Gazquez et al., 2017), so that those ELF data in different EQ-prone places in the globe will be utilized for monitoring pre-EQ effects in future to accumulate more anomalous SR data. Especially the simultaneous observation of vertical electric and horizontal magnetic field components, would be of the greatest importance in inferring the spatial structure of seismogenic ionospheric perturbations. This would provide us with a more hint to elucidate the process of lithosphere-atmosphere-ionosphere coupling, which is the greatest concern of seismoelectromagnetic scientists.

\section{Reference}

Bliokh, P. V., A. P. Nickolaenko, and Y. F. Filippov (1980), Global Electromagnetic Resonances in the Earth-ionosphere Cavity, Peter Perigrinus, New York, 168p.

Bliokh, P.V., Yu. P. Galuk, E.M. Hynninen, A.P. Nickolaenko, and L.M. Rabinowicz (1977), Resonance effects in the Earth-ionosphere cavity, Radiophysics and Quantum Electronics, 20, No.4, 339 - 345, https://doi.org/10.1007/BF01033918.

Christofilakis, V., G. Tatsis, C. Votis, I. Contopoloulos, C. Repapis, and V. Tritakis (2019), Significant ELF perturbations in the Schumann resonance band before and during a shallow mid-magnitude seismic activity in the Greek area (Kalpaki), J. Atmos. Solar-Terr. Phys., 182, 138-146.

Dobrovolsky, I.P., S. I. Zubkov, and V. L. Miachkin (1979). Estimation of the size of earthquake preparation zones, Pageoph., 117, 1025 - 1044. 
Florios, K., I. Contopoulos, V. Christofilakis, G. Tatsis, S. Chronopoulos, C. Repapis, and V. Tritakis (2019), Pre-seismic electromagnetic perturbations in two earthquakes in northern Greece, Pure Appl. Geophys., 2019, 1-13; doi:10.1007/s00024-019-02362-6.

Freund, F. (2013), Earthquake forewarning- A multidisciplinary challenge from the ground up to space, Acta Gecphysica, vol. 61, no.4, 775-807.

Greifinger, C., and P. Greifinger (1978), Approximate method for determining ELF eigen-values in the Earth-ionosphere waveguide, Radio Sci., vol. 13, 831-837.

Galuk, Yu.P., A. P. Nickolaenko, M. Hayakawa (2015), Comparison of exact and approximate solutions of the Schumann resonance problem for the knee conductivity profile, Telecommun. Radio Eng., vol. 74 (15), 1377-1390.

Galuk, Y.P., I.G. Kudintseva, A.P. Nickolaenko, and M. Hayakawa (2019), Scattering of ELF radio waves by a localized non-uniformity in the lower ionosphere, J. Atmos. Solar-Terr. Phys., vol. 194, 105093, https://doi.org/10.1016/j.jastp.2019.105093.

Gazquez, J. A., R. M. Garcia, N. N. Castellano, M. Fernandez-Ros, A. J. Perea-Moreno, and F. MazanoAgugliaro (2017), Applied engineering using Schumann resonance for earthquake monitoring, Appl. Sciences, vol. 7, 1113; doi:10.3390/app7111113.

Hayakawa, M., and Y. Hobara (2010), Current status of seismo-electromagnetics for short-term earthquake prediction, Geomatics, Natural Hazards Risk, vol. 1 (2), 115-155.

Hayakawa, M. (2015), Earthquake Prediction with Radio Techniques, John Wiley \& Sons, Singapore, $294 \mathrm{p}$.

Hayakawa, M., O. A. Molchanov, T. Ondoh, and E. Kawai (1996), The precursory signature effect of the Kobe earthquake on VLF subionospheric signals, J. Comm. Res. Lab. Tokyo, vol. 43, 169-180.

Hayakawa, M., K. Ohta, A. P. Nickolaenko, and Y. Ando (2005), Anomalous effect in Schumann resonance phenomena observed in Japan, possibly associated with the Chi-chi earthquake in Taiwan, Ann. Geophysicae, vol. 23, 1335-1346.

Hayakawa, M., A. P. Nickolaenko, M. Sekiguchi, K. Yamashita, Y. Ida, and M. Yano (2008), Anomalous ELF phenomena in the Schumann resonance band as observed at Moshiri (Japan) in possible association with an earthquake in Taiwan, Natural Hazards Earth Syst. Sci., vol. 8, 1309-1316.

Hayakawa, M., K. Ohta, V. M. Sorokin, A.K. Yaschenko, J. Izutsu, Y. Hobara and A. P. Nickolaenko (2010), Interpretation in terms of gyrotropic waves of Schumann resonance-like line emissions observed at Nakatsugawa in possible association with nearby Japanese earthquakes, J. Atmos. Solar-Terr. Phys., vol. 72, 1292-1298. 
Hayakawa, M., A. Schekotov, J. Izutsu, and A.P. Nickolaenko (2020), Seismogenic effects in ULF/ELF/VLF electromagnetic waves, Int. J. Electronics Appl. Res.(IJEAR), vol. 7 (in press).

Hayakawa, M., Y. Kasahara, T. Nakamura, Y. Hobara, A. Rozhnoi, M. Solovieva, O. A. Molchanov, and V. Korepanov (2011), Atmospheric gravity waves as a possible candidate for seismo-ionospheric perturbations, J. Atmos. Electr., vol. 31, no. 2, 129-140.

Heckman, S., E. Williams, and B. Boldi (1998), Total global lightning distribution revealed from Schumann resonance measurements, J. Geophys. Res., Atmospheres, vol. 103, 31775-31779.

Kudintseva, I.G., A.P. Nickolaenko, M.J. Rycroft, and A. Odzimek (2016). AC and DC global electric circuit properties and the height profile of atmospheric conductivity, Annals of Geophysics, vol. 59, 5, A0545; doi:10.4401/ag-6870.

Lizunov, G., T. Skorokhod, M. Hayakawa, and V. Korepanov (2020), Formation of ionospheric precursors of earthquakes- Probable mechanism and its substantiation, Open J. Earthquake Research (OJER), vol. 9, 142-169, doi:10.4236/ojer.2020.92009.

Maki, K., and T. Ogawa (1983), ELF emissions associated with earthquakes, Res. Lett. Atmospheric Electricity, vol. 13, 549-552.

Molchanov, O. A., and M. Hayakawa (2008), Seismo Electromagnetics and Related Phenomena: History and latest results, TERRAPUB, Tokyo, 189p.

Mushtak, V. C., and E. R. Williams (2002), ELF propagation parameters for uniform models of the Earthionosphere waveguide, J. Atmos. Solar-Terr. Phys., vol. 64, 1989-2001.

Nickolaenko, A. P. and M. Hayakawa (2002), Resonances in the Earth-Ionosphere Cavity, Kluwer Acad. Pub., Dordrecht, 380p.

Nickolaenko, A. P., and M. Hayakawa (2014), Schumann Resonances for Tyros : Essentials of Global Electromagnetic Resonance in the Earth-Ionosphere Cavity, Springer, Tokyo, 348p.

Nickolaenko A.P., A. V. Shvets, and M. Hayakawa (2016), Propagation of Extremely Low Frequency Radio Waves, Ed. by J. Webster, Wiley Encyclopedia of Electrical and Electronics Engineering. Hoboken, USA: John Wiley \& Sons, Inc., pp. $1-20$ https://doi.org/10.1002/047134608X.W1257.pub2,eeee@wiley.com].

Nickolaenko A.P., Yu. P. Galuk, and M. Hayakawa (2017), Extremely low frequency (ELF) wave propagation: Vertical profile of atmospheric conductivity matching with Schumann resonance data, Horizons in World Physics, vol. 288, Ed. by Albert Reimer, Chapter 6, pp. 105 - 128, Nova Science Pub.,Inc., New York, 2017, ISBN: 978-1-63485-882-3. 
Nickolaenko, A. P., Y. P. Galuk, and M. Hayakawa (2018a), The effect of a compact ionosphere disturbance over the earthquake: A Focus on Schumann resonance, Int. J. Electronics Appl. Res. (IJEAR), vol. 5, issue 2, 11-39. http://eses.net.in/online journal.html

Nickolaenko, A. P., Y. P. Galuk, and M. Hayakawa (2018b), Source bearing of extremely low frequency waves in the Earth-ionosphere cavity with day-night nonuniformity, J. Geophys. Res., Atmospheres, vol. $123,10895-10910$.

Nickolaenko, A. P., M. Hayakawa, M. Sekiguchi, Y. Ando, and K. Ohta (2006), Model modifications in Schumann resonance intensity caused by a localized ionosphere disturbance over the earthquake epicenter, Ann. Geophysicae, vol. 24, 567-575.

Nickolaenko, A. P., E. I. Yatsevich, A. V. Shvets, M. Hayakawa, and Y. Hobara (2011), Universal and local time variations deduced from simultaneous Schumann resonance records at three widely spaced observatories, Radio Sci., vol. 46, RS5003, doi:10.129/2011RS004663.

Ohta, K., N. Watanabe, and M. Hayakawa (2006), Survey of anomalous Schumann resonance phenomena observed in Japan, in possible association with earthquakes in Taiwan, Phys. Chem. Earth, vol. 31, $397-$ 402.

Ohta, K., J. Izutsu, and M. Hayakawa (2009), Anomalous excitation of Schumann resonances and additional anomalous resonances before the 2004 Mid-Niigata prefecture earthquake and the 2007 Noto Hantou earthquake, Phys. Chem. Earth, vol. 34, 441-448.

Ohta, K., J. Izutsu, A. Schekotov, and M. Hayakawa (2013), The ULF/ELF electromagnetic radiation before the 11 March 2011 Japanese earthquake, Radio Sci., vol. 48, 589-596, doi:10.1002/ rds.20064.

Ouzounov, D., S. Pulinets, K. Hattori, and P. Taylor (Eds) (2018), Pre-Earthquake Processes: A multidisciplinary approached to earthquake prediction studies, American Geophysical Union, Geophysical Monograph 234, Wiley, 365p.

Ouyang, X., X. Zhang, A. P. Nickolaenko, M. Hayakawa, X. Shen, and Y. Miao (2013), Schumann resonance observation in China and anomalous disturbance possibly associated with the Tohoku M9.0 earthquake, Earthquake Sci., vol. 26, 137-145, doi:10.1007/s11589-013-0009-0.

Pracser, E., T. Bozoki, G. Satori, E. Williams, A. Guha, and H. Yu (2019), Reconstruction of global lightning activity based on Schumann resonance measurements: Model and synthetic tests, Radio Sci., vol. 54, 254-267.

Price, C., O. Pechony, and E. Greeenberg (2007), Schumann resonance in lightning research, J. Lightning Res., vol. 1, 1-15.

Pulinets, S., and K. Boyarchuk (2004), Ionospheric Precursors of Earthquakes, Springer, Berlin, 315p. 
Pulinets, S., and D. Ouzounov (2011), Lithosphere-atmosphere-ionosphere coupling (LAIC) model- A unified concept for earthquake precursors validation, J. Asian Earth Sci., vol. 41, 371-382.

Ruhzin, Y. Y., and A. K. Depueva (1996), Seismoprecursors in space as plasma and wave anomalies, J. Atmos. Electr., vol. 16, no. 3, 271-288.

Samarskii, A.A. (2001), The Theory of Difference Schemes, Marcel Dekker, Inc, New York - Basel, $761 p$.

Satori, G., V. Mushtak, and E. Williams (2009), Schumann resonance signatures of global lightning activity, in "Lightning: Principles, instruments, and applications", Ed. by H. D. Betz, U. Schumann, and P. Laroche, Springer Dordrecht, 347-386.

Schekotov, A. Y., O. A. Molchanov, M. Hayakawa, E. N. Fedorov, V. N. Chebrov, V. I. Sinitsin, E. E. Gordeev, G. G. Belyaev, and N. V. Yagova (2007), ULF/ELF magnetic field variations from atmosphere by seismicity, Radio Sci., vol. 42, RS6S90, doi:10.1029/2005RS003441.

Schekotov, A., and M. Hayakawa (2017), ULF/ELF Electromagnetic Phenomena for Short-term Earthquake Prediction, LAP Lambert Academic Publishing, Mauritius, 102p. doi:10.1029/2005RS003441.

Schekotov, A., E. Fedorov, O. A. Molchanov, and M. Hayakawa (2013), Low frequency electromagnetic precursors as a prospect for earthquake prediction, in "Earthquake Prediction Studies: Sesimo Electromagnetics", Ed. by M. Hayakawa, TERRAPUB Tokyo, 81-99.

Sentman, D. D. (1995), Schumann resonances, in "Handbook of Atmospheric Electrodynamics", Ed. by H. Volland, vol. 1, CRC Press, Boca Raton, 267-298.

Sekiguchi, M., M. Hayakawa, A. P. Nickolaenko, and Y. Hobara (2006), Evidence of a link between the intensity of Schumann resonance and global surface temperature, Ann. Geophysicae, vol. 24, 1597315984.

Shvets, A., and M. Hayakawa (2011), Global lightning activity on the basis of inversions of natural ELF electromagnetic data observed at multiple stations around the world, Survey Geophys., vol. 32, issue 6, 705-732.

Sorokin, V., and M. Hayakawa (2008), On the generation of narrow-banded ULF/ELF pulsations in the lower ionospheric conducting layer, J. Geophys. Res., 113, A06306. https://doi.org/10.1029/JA013094.

Sorokin, V., V. Chmyrev, and M. Hayakawa (2015), Electrodynamic Coupling of LithosphereAtmosphere-Ionosphere of the Earth, Nova Science Pub. Inc., New York, 326p. 
Sorokin, V. M., V. Chmyrev, and M. Hayakawa (2020), A review on electrodynamic influence of atmospheric processes to the ionosphere, Open J. Earthquake Research (OJER), vol. 9, 113-141, doi:10.4236/ojer.2020.92008.

Surkov, V., and M. Hayakawa (2014), Ultra and Extremely Low Frequency Electromagnetic Fields, Springer Tokyo, 486p.

Uyeda. S., T. Nagao, and M. Kamogawa (2008), Short- term earthquake prediction: Current status of seismo-electromagnetics, Tectonophysics, vol. 470, 205-213.

Wait, J. R. (1970), Electromagnetic Waves in Stratified Media, Pergamon Press, Oxford, 608p.

Williams, E. R. (1992), The Schumann resonance: a global tropical thermometer, Science, vol. 256, 11841188.

Yang, S. S., T. Asano, and M. Hayakawa (2019), Abnormal gravity wave activity in the stratosphere prior to the 2016 Kumamoto earthquakes, J. Geophys. Res., Space Phys., vol. 124, https://doi.org/10.1029/2018JA026002.

Yang, S. S., and M. Hayakawa (2020), Gravity wave activity in the stratosphere before the 2011 Tohoku earthquake as the mechanism of lithosphere-atmosphere-ionosphere coupling, Entropy, vol. 22, 110; doi:10.3390/e22010110.

Yoshida, M., T. Yamauchi, T. Horie, and M. Hayakawa (2008), On the generation mechanism of terminator times in subionospheric VLF/LF propagation and its possible application to seismogenic effects, Natural Hazards Earth Syst. Sci., vol. 8, 129-134. 